\title{
GNSS DOPPLER VELOCITY BASED ON ADAPTIVE ROBUST KALMAN FILTERING
}

\author{
LIU Hui ${ }^{1}$, XIN Mingzhen ${ }^{1}$, WEI Jinjin ${ }^{1}$, LIANG Yuke ${ }^{1}$, YANG Fanlin ${ }^{1,2}$ \\ ${ }^{1}$ College of Geomatics, Shandong University of Science and Technology, Qingdao 266590, China-(liu,xin,wei,yang) \\ @ http://gc.sdust.edu.cn/; \\ ${ }^{2}$ Key Laboratory of Surveying and Mapping Technology on Island and Reef, National Administration of Surveying, Mapping and \\ Geoinformation, Qingdao 266590, China
}

KEY WORDS: Out Doppler Velocity, Adaptive Robust Kalman Filter, PDOP, IGG-III, Adaptive Factor

\begin{abstract}
The main factors affecting the error of Doppler velocity measurement mainly come from the measurement errors of GNSS data, influence of different motion states on GNSS velocity measurement and the noise of different receiver types. To improve the precision of GNSS velocity estimation, an algorithm of adaptive robust Kalman filter based on the PDOP was put forward. PDOP value as well as the number of satellite in each epoch are used as a criterion in the velocity processing. While the PDOP value is greater than the threshold value, which means the observation accuracy is low, then the robust Kalman filter based on IGG - III scheme is introduced. While the PDOP value is between the threshold values, which means the observation precision is normal, adaptive factor could be determined normally, and the single-factor three-stage adaptive model is applied for Kalman filtering. If the above two conditions are not consistent, it indicates that the prediction accuracy of the local epoch satellite is high, and Kalman filtering can be directly used. Through the experiment of shipborne GNSS velocity measurement, it was proved that comparing with conventional least square, the algorithm based on the adaptive robust Kalman filtering can improve the accuracy and stability of the GNSS velocity determination.
\end{abstract}

\section{INTRODUCTION}

Velocity based on the position difference, Doppler, carrier-phase derived Doppler are the three common ways of the velocity estimation.position difference mainly using precise coordinates of GNSS with adjacent epoch, the original Doppler calculated the instantaneous GNSS velocity, which related to the type of receiver, and the out Doppler is derived by carrier phase observations in adjacent epoch with high precision, but which need to eliminate the influence of the cycle slip(Zheng ,Tang, 2015). Kalman filtering is the most commonly used in GNSS position and velocity estimation (Wang, Liang, 2018; Li et al. 2018). In theory, as the grown in quantity of observation data, more accurate valuation could be obtained by the state of the Kalman filter. But sometimes the noise of GNSS dynamic motion may not be able to accurately model or is not a normal distribution, so the accuracy of the results of using Kalman filter for dynamic calculating may be affected, which lead to larger error between actual state and the state. At the same time, the filtering divergence phenomenon happens (Gao S et al. 2011).

In order to solve the above problem, scholars have successively put forward the adaptive Kalman filter algorithm and apply it in dynamic navigation and positioning. A new adaptive differential filtering theory was established (Yang et al. 2001; Yang et al. 2001), which applies the principle of differential resistance estimation to control abnormal observations, and introduces the adaptive factor to control the effect of the error in dynamic model. Four kinds of error learning statistics were successively constructed, namely, state mismatch statistics (Yang et al. 2001; Yang et al. 2001), prediction residual statistics (Yang ,Gao,2006), the variance component ratio statistics based on observation information and dynamic model prediction information (Yang,Xu,2003), and the mismatch statistics based prediction velocity model and calculation velocity (Cui ,Yang,2006).Four kinds of adaptive factors were established, namely three-segment function model (Yang et al.2001), two-segment function model (Yang et al. 2001), exponential function model (Yang, Gao,2005) and weight function model (Ren et al. 2005). When multi-factor becomes single factor, multi-factor adaptive filtering is single factor adaptive filtering. While only the position parameter factor and velocity factor are included in the multi-factor, the multi-factor adaptive filter becomes the classification factor adaptive filter. Adaptive robust Kalman filtering can effectively restrain the influence of the error in abnormal observation and error in dynamic model. Based on this, an adaptive Kalman filtering 
algorithm for out Doppler velocity based on single point station was put forward. the method select the resistance model and adaptive model based on the PDOP value and the number of the satellite in each epoch, using IGGIII estimation scheme to control the effect of abnormal observations, at the same time, using the single-factor three-stage adaptive model to adjust the proportion between the covariance matrix of the predicted value and the covariance matrix of state vector. In order to test the accuracy of the algorithm, through a set of shipborne GNSS/INS dynamic test data, the accuracy of the velocity filtering method and the least squares velocity measurement in this paper were compared and analyzed.

\subsection{Principle of GPS single point velocity measurement GNSS}

Pseudo-range difference is a widely used differential positioning technology. Its principle is to set up a reference station on a known coordinate point, calculate the true distance between the reference station and the satellite through the known coordinate, and then make a difference between the calculated true distance and the observed pseudo-distance to obtain pseudo-distance correction and send it to the user for correction.

Pseudo-range observation equation (XU et al. 2016):

$$
\begin{aligned}
\tilde{\rho}_{i}^{j}\left(t_{i}\right)= & \rho_{i}^{j}\left(t_{i}\right)+c\left(\delta t^{s}\left(t_{i}\right)-\delta t^{r}\left(t_{i}\right)\right)-\delta_{\text {ion }}\left(t_{i}\right)+\delta_{\text {trop }}\left(t_{i}\right) \\
& +\delta_{r e l}\left(t_{i}\right)+\varepsilon_{p}\left(t_{i}\right)
\end{aligned}
$$

Deriving the pseud-orange observation equation:

$$
\begin{aligned}
\dot{\tilde{\rho}}_{i}^{j}\left(t_{i}\right)= & \dot{\rho}_{i}^{j}\left(t_{i}\right)+c\left(\delta \dot{t}^{s}\left(t_{i}\right)-\delta \dot{t}^{r}\left(t_{i}\right)\right)-\dot{\delta}_{\text {ion }}\left(t_{i}\right)+\dot{\delta}_{\text {trop }}\left(t_{i}\right) \\
& +\dot{\delta}_{r e l}\left(t_{i}\right)+\dot{\varepsilon}_{p}\left(t_{i}\right)
\end{aligned}
$$

The out Doppler is relative to the original Doppler, which is the Doppler value derived from the pseudo range, carrier phase and Doppler relationship provided in the observation file.

At the moment $t_{i}$, the pseud orange observation equation between the station $r$ and the satellite $S$ is differentiated (XU et al. 2016):

$$
\begin{aligned}
\lambda D\left(t_{i}\right)= & \dot{R}_{r}^{s}\left(t_{i}\right)+c\left(\delta \dot{t}^{s}\left(t_{i}\right)-\delta t^{r}\left(t_{i}\right)\right)-\dot{\delta}_{\text {ion }}\left(t_{i}\right)+\dot{\delta}_{\text {trop }}\left(t_{i}\right) \\
& +\dot{\delta}_{r e l}\left(t_{i}\right)+\dot{\varepsilon}_{p}\left(t_{i}\right)
\end{aligned}
$$

In the formula, $\lambda$ is the carrier wavelength; $D\left(t_{i}\right)$ is out Doppler observation, each "." term is the time variability of each corresponding variable; the $\dot{R}_{r}^{s}\left(t_{i}\right)$ is the geometric distance between the $t_{i}$ time satellite and the receiver. $\delta_{\text {trop }}\left(t_{i}\right), \delta_{\text {ion }}\left(t_{i}\right)$ is the tropospheric and ionospheric in carrier phase observation at the $t_{i}$ time. The $\dot{\delta}_{r e l}\left(t_{i}\right)$ is the effect of the relativistic effect in the $t_{i}$ time, $\varepsilon_{p}\left(t_{i}\right)$ means other observed noise.

Out Doppler observations using the adjacent epoch differential structure can effectively eliminate the ambiguity parameters. Using the basic principle of the difference between the epochs before and after the phase, the out Doppler can be expressed as ( He, 2015)

$$
\dot{\phi}=\frac{\phi\left(t_{i+1}\right)-\phi\left(t_{i}\right)}{\Delta t}=\tilde{D}\left(t_{i+1}\right)
$$

In the formula, $\phi\left(t_{i}\right) 、 \phi\left(t_{i+1}\right)$ is the phase observation values of the previous epoch time and the last epoch time $t+\Delta t$, and $\Delta t$ means for the sampling interval.

\subsection{Kalman filtering}

Kalman filtering is divided into the following two processes (Yang, Gao, 2006)

(1) Time upate:

$$
\begin{aligned}
& \hat{X}_{k, \mathrm{k}-1}=\Phi_{k, k-1} \hat{X}_{k-1}+A U \\
& P_{k, k-1}=\Phi_{k, k-1} P_{k-1} \Phi_{k, k-1}^{T}+Q_{k-1}
\end{aligned}
$$

(2) Measurement update

$$
\begin{gathered}
K_{k}=P_{k, k-1} H_{k}^{T}\left(H_{k} P_{k, k-1} H_{k}^{T}+R_{k}\right)^{-1} \\
\hat{X}_{k}=\hat{X}_{k, k-1}+K_{k}\left(Z_{k}-H_{k} \hat{X}_{k, k-1}\right) \\
P_{k}=\left(I-K_{k} H_{k}\right) P_{k, k-1}
\end{gathered}
$$

$K_{k}$ is the filter gain matrix; $P_{k, k-1}$ is the covariance matrix of the state vector in $t_{k-1}$ time

The state quantity includes the position, velocity, acceleration component of the user's motion state, and the clock difference and clock drift of the user's clock error.

$$
\begin{aligned}
& X=\left[\begin{array}{ll}
X_{s} & X_{T}
\end{array}\right]^{T} \\
& X_{s}=\left(\begin{array}{lllllllll}
x & x_{v} & x_{a} & y & y_{v} & y_{a} & z & z_{v} & z_{a}
\end{array}\right)^{T} \\
& X_{T}=\left(\begin{array}{llll}
\tau_{1} & \dot{\tau}_{1} & \tau_{2} & \dot{\tau}_{2}
\end{array}\right)^{T}
\end{aligned}
$$


While $X_{s}$ denote the vector of the receiver displacement, velocity and acceleration, respectively, $X_{T}$ means the vector of the receiver clock and receiver clock drift.

According to the basic principle of the current statistical model, the state can be expressed as (Yang,Gao,2006):

$$
\begin{gathered}
\hat{X}_{S(k, \mathrm{k}-1)}=\Phi_{S(k, k-1)} \hat{X}_{S(k-1)}+W_{S(k-1)} \\
\Phi_{S(k, k-1)}=\left[\begin{array}{ccc}
\Phi_{1 x} & 0_{3 \times 3} & 0_{3 \times 3} \\
0_{3 \times 3} & \Phi_{1 y} & 0_{3 \times 3} \\
0_{3 \times 3} & 0_{3 \times 3} & \Phi_{1 z}
\end{array}\right] \quad \Phi_{1 x}=\Phi_{1 y}=\Phi_{1 z}=\left[\begin{array}{ccc}
1 & T & \frac{T^{2}}{2} \\
0 & 1 & T \\
0 & 0 & 1
\end{array}\right] \\
\hat{X}_{\tau(k, \mathrm{k}-1)}=\left[\begin{array}{cccc}
1 & T & 0 & 0 \\
0 & 1 & 0 & 0 \\
0 & 0 & 1 & T \\
0 & 0 & 0 & 1
\end{array}\right] \hat{X}_{\tau(k-1)}+W_{\tau(k-1)}
\end{gathered}
$$

the Observation equation construction are given by

$$
\begin{gathered}
Y_{k}=H_{k} X_{k}+Z_{k} \\
Y=\left[\begin{array}{llllllllll}
\rho_{1} & \dot{\rho}_{1} & \rho_{2} & \dot{\rho}_{2} & \rho_{3} & \dot{\rho}_{3} & \cdots & \cdots & \rho_{n} & \dot{\rho}_{n}
\end{array}\right]^{T}
\end{gathered}
$$

While $\rho_{n}, \dot{\rho}_{n}$ are the observed precision code and out Doppler observations from a satellite to the receiver, $H_{k}$ is Observation matrix, $X_{k}$ is State quantity $Z_{k}$ is the measurement noise.

$$
\begin{aligned}
& \rho_{1}=\sqrt{\left(x-x_{s 1}\right)^{2}+\left(y-y_{s 1}\right)^{2}+\left(z-z_{s 1}\right)^{2}}+c \tau_{1} \\
& \rho_{2}=\sqrt{\left(x-x_{s 2}\right)^{2}+\left(y-y_{s 2}\right)^{2}+\left(z-z_{s 2}\right)^{2}}+c \tau_{1} \\
& \rho_{3}=\sqrt{\left(x-x_{s 3}\right)^{2}+\left(y-y_{s 3}\right)^{2}+\left(z-z_{s 3}\right)^{2}}+c \tau_{2} \\
& \rho_{4}=\sqrt{\left(x-x_{s 4}\right)^{2}+\left(y-y_{s 4}\right)^{2}+\left(z-z_{s 4}\right)^{2}}+c \tau_{2} \\
& \ldots \\
& \dot{\rho}_{1}=\left(\left(x-x_{s 1}\right)\left(x_{v}-x_{v 1}\right)+\left(y-y_{s 1}\right)\left(y_{v}-y_{v 1}\right)+\left(z-z_{s 1}\right)\left(z_{v}-z_{v 1}\right)\right) / \rho_{1}+c \dot{c}_{1} \\
& \dot{\rho}_{2}=\left(\left(x-x_{s 2}\right)\left(x_{v}-x_{v 2}\right)+\left(y-y_{s 2}\right)\left(y_{v}-y_{v 2}\right)+\left(z-z_{s 2}\right)\left(z_{v}-z_{v 2}\right)\right) / \rho_{2}+c \dot{\tau}_{1} \\
& \dot{\rho}_{3}=\left(\left(x-x_{s 3}\right)\left(x_{v}-x_{v 3}\right)+\left(y-y_{s 1}\right)\left(y_{v}-y_{v 3}\right)+\left(z-z_{s 3}\right)\left(z_{v}-z_{v 3}\right)\right) / \rho_{3}+c \dot{\tau}_{2} \\
& \dot{\rho}_{4}=\left(\left(x-x_{s 4}\right)\left(x_{v}-x_{v 4}\right)+\left(y-y_{s 1}\right)\left(y_{v}-y_{v 4}\right)+\left(z-z_{s 4}\right)\left(z_{v}-z_{v 4}\right)\right) / \rho_{4}+c \dot{\tau}_{2} \\
& H_{k}=\left[\begin{array}{llllll}
H_{1} & H_{2} & H_{3} & H_{4} & \cdots & H_{n}
\end{array}\right]^{T} \\
& H_{i}=\left[\begin{array}{ccccccccccccc}
h_{i x} & 0 & 0 & h_{i y} & 0 & 0 & h_{i z} & 0 & 0 & 1 & 0 & 0 & 0 \\
0 & h_{i x} & 0 & 0 & h_{i x} & 0 & 0 & h_{i x} & 0 & 0 & 1 & 0 & 0
\end{array}\right], i=G P S(1,2 \cdots) \\
& H_{i}=\left[\begin{array}{ccccccccccccc}
h_{i x} & 0 & 0 & h_{i y} & 0 & 0 & h_{i z} & 0 & 0 & 0 & 0 & 1 & 0 \\
0 & h_{i x} & 0 & 0 & h_{i x} & 0 & 0 & h_{i x} & 0 & 0 & 0 & 0 & 1
\end{array}\right], i=B D S(3,4 \cdots)
\end{aligned}
$$

$$
\begin{aligned}
& h_{i x}=\frac{\hat{x}_{k / k-1}-x_{s 1}}{D_{i}}, h_{i y}=\frac{\hat{y}_{k / k-1}-y_{s 1}}{D_{i}}, h_{i z}=\frac{\hat{z}_{k / k-1}-z_{s 1}}{D_{i}}(i=1,2,3, \cdots n) \\
& D_{i}=\sqrt{\left(\hat{x}_{k / k-1}-x_{s 1}\right)^{2}+\left(\hat{y}_{k / k-1}-y_{s 1}\right)^{2}+\left(\hat{z}_{k / k-1}-z_{s 1}\right)^{2}}
\end{aligned}
$$

From the above filters, we can see that in addition to establishing the state model and observation model of the system, the following problems need to be solved in solving user state (Yang et al. 2001): setting of initial state $\mathrm{X}$ and error covariance matrix $P$, setting of process noise variance matrix $Q$, setting of observation noise variance matrix R. Regarding the setting of $X$, the least square method is used to provide the initial state quantity.

The process noise $\mathrm{w}$ of linear discrete stochastic systems obeys the Gauss-Markon process, and the initial value of $\mathrm{Q}$ is determined by the lower value (Tu, 2014).

$$
Q_{k}=\left[\begin{array}{ccccccc}
\frac{\tau^{5} \cdot p d_{a}^{2} \cdot I}{20} & \frac{\tau^{4} \cdot p d_{a}^{2} \cdot I}{8} & \frac{\tau^{3} \cdot p d_{a}^{2} \cdot I}{6} & 0 & 0 & 0 & 0 \\
\frac{\tau^{4} \cdot p d_{a}^{2} \cdot I}{8} & \frac{\tau^{3} \cdot p d_{a}^{2} \cdot I}{3} & \frac{\tau^{2} \cdot p d_{a}^{2} \cdot I}{2} & 0 & 0 & 0 & 0 \\
\frac{\tau^{3} \cdot p d_{a}^{2} \cdot I}{6} & \frac{\tau^{2} \cdot p d_{a}^{2} \cdot I}{2} & \tau^{2} \cdot p d_{a}^{2} \cdot I & 0 & 0 & 0 & 0 \\
0 & 0 & 0 & \tau \cdot p d_{d_{1}}^{2} & 0 & 0 & 0 \\
0 & 0 & 0 & 0 & \tau \cdot p d_{f_{1}}^{2} & 0 & 0 \\
0 & 0 & 0 & 0 & 0 & \tau \cdot p d_{d_{2}}^{2} & 0 \\
0 & 0 & 0 & 0 & 0 & 0 & \tau \cdot p d_{f_{2}}^{2}
\end{array}\right]
$$

Where $\tau$ is the sampling rate, $I$ is a $3 \times 3$ identity matrix, $p d_{a}, p d_{d t}, p d_{f}$ are the power density of the acceleration, receiver clock noises and noises of Clock drift. respectively. Normally, $p d_{a}$ can be set as a few $\mathrm{cm} \mathrm{s}-5 / 2$, and $p d_{d t}$ is set to white noise with a very large value (100ms-1/2). (Yang et al. 2001).

Observed noise variance matrix:

$$
R_{k}=\left[\begin{array}{cc}
\sigma_{P}^{2} & 0 \\
0 & \sigma_{D}^{2}
\end{array}\right]
$$

The variance matrix of pseudo-distance observations and out Doppler:

$$
\begin{aligned}
& \sigma_{P}^{2}=\operatorname{diag}\left(\sigma_{P_{1}}^{2} \quad \sigma_{P_{2}}^{2} \quad \sigma_{P_{3}}^{2} \quad \cdots \quad \sigma_{P_{n}}^{2}\right) \\
& \sigma_{D}^{2}=\operatorname{diag}\left(\begin{array}{lllll}
\sigma_{D_{1}}^{2} & \sigma_{D_{2}}^{2} & \sigma_{D_{3}}^{2} & \cdots & \sigma_{D_{n}}^{2}
\end{array}\right)
\end{aligned}
$$

$\sigma_{p}^{2}, \sigma_{D}^{2}$ Is also a $n \times n$ diagonal matrix. 


\subsection{Adaptive robust Kalman filtering}

Corresponding to the standard Kalman filter model, the rewritten gain matrix and the PDOP (Phillips et al. 1984) is as follows:

$$
\begin{gathered}
K_{k}=\left(H_{k}^{T} \bar{P}_{k} H_{k}+\bar{P}_{x k}\right)^{-1} H_{k}^{T} \bar{P}_{k} \\
Q_{x x}=H^{T} H \\
P D O P=\sqrt{\left(\sigma_{x}^{2}+\sigma_{y}^{2}+\sigma_{z}^{2}\right)}
\end{gathered}
$$

where $\bar{P}_{k}$ is robust equivalence weights, $\bar{P}_{x k}$ denotes the adaptive equivalence weights .

Focusing on the gain matrix, an adaptive robust Kalman model based on PDOP(Position Dilution of Precision) and the number was proposed. Case1: When the PDOP is greater than the threshold value, it is considered that the observation accuracy is low, and the IGG-III robust model is used for Kalman filter smoothing. Case 2: When the PDOP is between the threshold value, it is considered that the measurement accuracy is normal, and the adaptive factor can be determined normally. Then the single-factor three-stage adaptive model is used to adjust the adaptive equivalence weights. Case 3: If neither of the above cases is met, standard Kalman filtering is used. The selection of threshold for Robust equivalence weights and adaptive equivalence weights are both based on the experience:

In this paper we set case1:

$$
\begin{aligned}
& P D O P>4 \| \text { satnum }<8 \|(P D O P>2.5 \& \& \\
& \text { satnum }<11) \|(P D O P>3.5 \& \& \text { satnum }<14)
\end{aligned}
$$

Case2:

$$
(P D O P<3 \& \& \text { satnum }>10) \|(P D O P<3.1
$$

$\& \&$ satnum $>11) \|(P D O P<2)$

\section{(1) IGG-III robust model:}

The IGGIII scheme (Galili et al. 1987) is the most widely used, and it divides the weight of measured values into three cases. the expression is :

$$
\left\{\begin{array}{l}
\bar{p}_{i}=p_{i},\left|v_{i}\right|<k_{0} \\
\bar{p}_{i}=p_{i} \bullet \frac{k_{0}}{\left|v_{i}\right|} \bullet\left(\frac{k_{1}-\left|v_{i}\right|}{k_{1}-k_{0}}\right)^{2}, k_{0} \leq\left|v_{i}\right|<k_{1} \\
\bar{p}_{i}=0, k_{1} \leq\left|v_{i}\right|
\end{array}\right.
$$

Where $k_{0}$ is the security point; $k_{1}$ is the elimination point; $p_{i}$ is the weight matrix of the observation; $v_{i}$ is the normalized residual of the $i$ observation; $\bar{P}_{i}$ is the weight after the $i$ observation is reduced. It can be seen from the above formula that the IGGIII scheme divides the residual value into three parts. When obeying the normal distribution, the weight of the observed value remains unchanged; if the observed value exceeds a certain range, the weighting process is performed; if individual observations appear obviously abnormal, the weight is 0 at this time. Therefore, the IGGIII program has the best resistance.

(2) Single-factor three-stage adaptive model: adaptive equivalence weights $\bar{P}_{x k}$ Can be expressed as:

$$
\bar{P}_{x k}=\alpha \cdot P_{x k}
$$

where $\alpha$ is adaptive factor,

The three-stage adaptive factor is constructed by referring to the IGGIII equivalent weight (Yang et al. 2001; Yang et al. 2006 ), which set the prediction residual value as a statistic, and the three-stage adaptive factor can be constructed as:

$$
\alpha_{k}=\left\{\begin{array}{cc}
1 & \left|\Delta \bar{V}_{k}\right| \leq c_{0} \\
\frac{c_{0}}{\mid \Delta \bar{V}_{k}\left(\frac{c_{1}-\left|\Delta \bar{V}_{k}\right|}{c_{1}-c_{0}}\right)^{2}} & c_{0}<\left|\Delta \bar{V}_{k}\right| \leq c_{1} \\
0 & \left|\Delta \bar{V}_{k}\right|>c
\end{array}\right.
$$

Where $c_{0}, c_{1}$ are also constants. Generally both can be set to $1.0 \sim 1.5$ or $3.0 \sim 8.5$ 


\section{Algorithm flow chart}

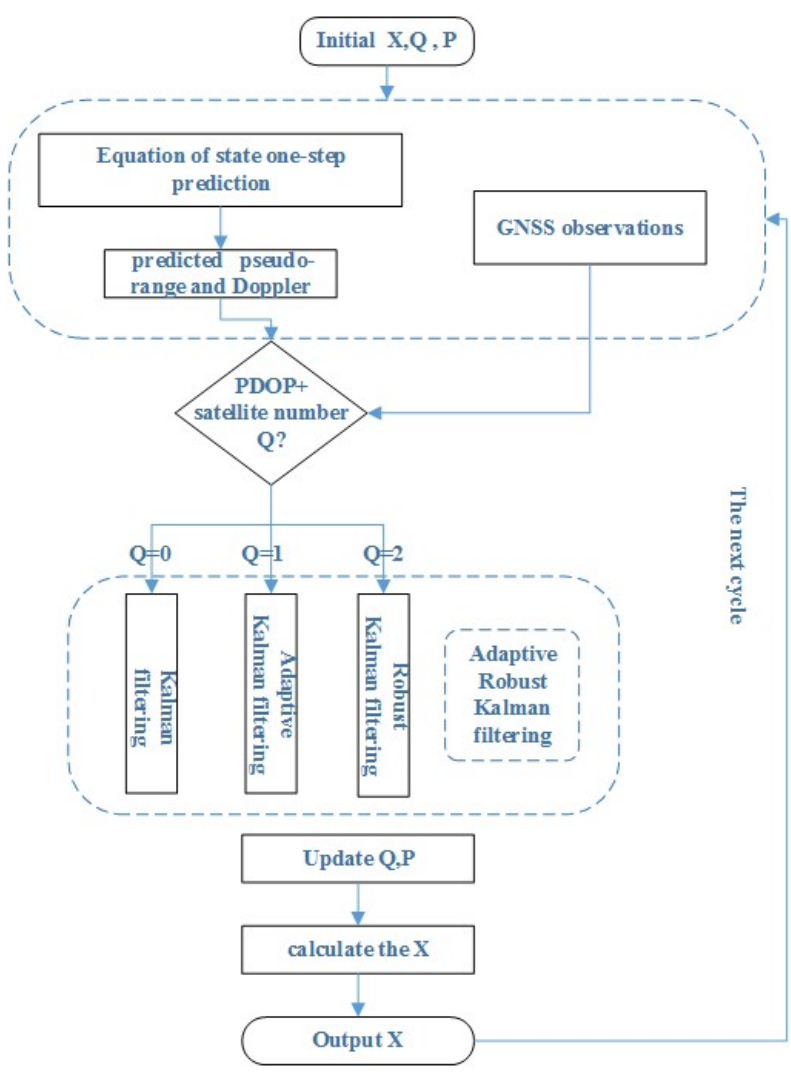

Figure 1. algorithm flow chart

\section{VALIDATION AND DISCUSSION}

\section{1 static test}

A set of GNSS receivers typed Propak6 was used to collect one hour of static data. The observation period was from April 30, 2015, 9:00-10:00, the sampling rate was $1 \mathrm{~Hz}$, and the satellite elevation angle was 15 degrees. The least square method and the adaptive robust Kalman method are used respectively to solve the carrier phase derived doppler velocity, If static observations were taken to calculate, the true value of the filter algorithm and the Least Squares method should be zero, and the solution of the calculated speed value could be considered the true error of speed algorithm.

Schm1: Least Squares( L-S)

Schm2: adaptive robust Kalman method (ARKF)

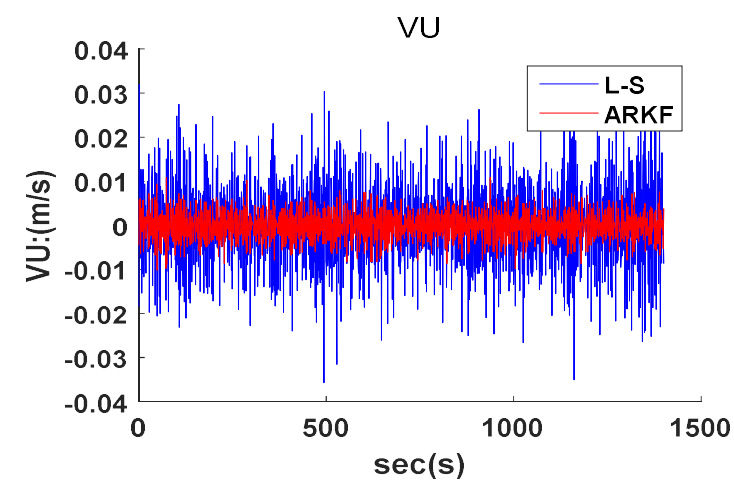

Figure 2. the velocity in $U$ direction measured by L-S and ARKF

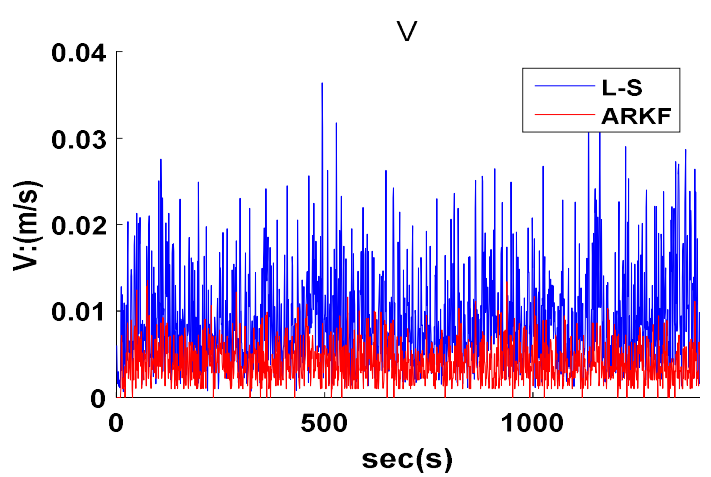

Figure 3 . the total velocity measured by L-S and ARKF

We can seen from the Fig.2 and Fig.3 that the true error of the ARKF methods is better than $1.00 \mathrm{~m} / \mathrm{s}$ in the $\mathrm{U}$ direction, and the RMS of the total speed is also better than $1.00 \mathrm{~cm} / \mathrm{s}$. The least squares method is inferior to the ARKF methods in both the $U$ direction and the total velocity. And there is an obvious systematic deviation of about $2.00 \mathrm{~cm} / \mathrm{s}$ in the direction of $U$ in the settlement velocity of LS method, and the oscillation is severe.

\begin{tabular}{lllll}
\hline $\mathrm{V}(\mathrm{m} / \mathrm{s})$ & $\max$ & $\min$ & mean & RMS \\
\hline $\mathrm{L}-\mathrm{S}$ & 0.0364 & 0 & 0.0091 & 0.0108 \\
\hline ARKF & 0.0136 & 0 & -0.0020 & 0.0047 \\
\hline
\end{tabular}

Table.1 Statistics of Velocity based on the L-S and ARKF

Tab. 1 shows the statistical results of the comparison between the total velocity calculated by L-S and ARKF methods. It can be clearly seen from the table that there is a big difference between the them: the maximum and average speed calculated by LS method are $3.64 \mathrm{~cm} / \mathrm{s}$ and $0.91 \mathrm{~cm} / \mathrm{s}$ respectively, while ARKF is $1.36 \mathrm{~cm} / \mathrm{s}$ and $-0.20 \mathrm{~cm} / \mathrm{s}$, and the residual of LS error is up to $1 \mathrm{~cm} / \mathrm{s}$, while $A R K F$ is $0.4 \mathrm{~cm} / \mathrm{s}$, which can verify that the ARKF method has better accuracy than L-S and has better denoising effect.

\section{2 kinematic test}

In order to verify the feasibility and accuracy of the ARKF 
algorithm, data of continuous acquisition for 1.5 hours was performed on May 31, 2017, using a shipborne GNSS-INS (HYDRINS) integrated navigation system in the Yangtze River. The type of kinemaic GNSS receiver is NovAtel GPSCard, the sampling frequency is $1 \mathrm{~Hz}$, the sampling frequency of the HYDRINS is $200 \mathrm{HZ}$, and a NovAtel GPSCard receiver is placed on the shore as a base station which sampling frequency is also $1 \mathrm{HZ}$ for simultaneous sampling(as shown in Fig. 4).

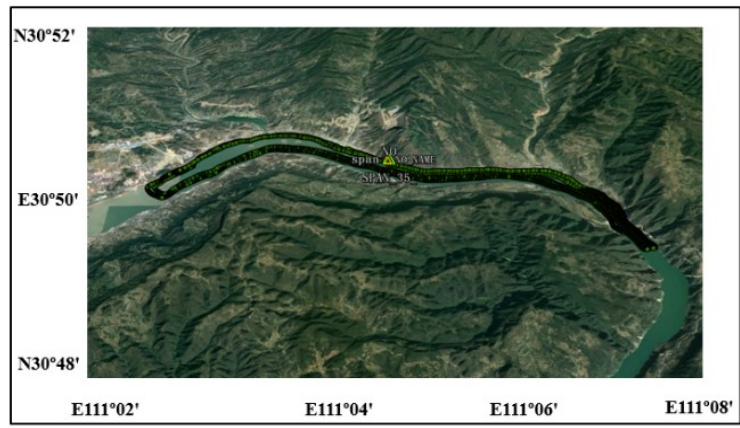

Figure 4. Topographic map of survey area
Since the tight combination mode of Internial Explorer (IE) software solves the velocity with an accuracy better than $0.2 \mathrm{~cm} / \mathrm{s}$, the IE velocity is used as the reference true value.

It can be seen from Fig. 5, that the trends of velocity in the $\mathrm{E}$ and $\mathrm{N}$ directions calculated by LS and ARKF method are substantially the same. The speed of the first 2500 epochs in E-direction is $-2 \mathrm{~m} / \mathrm{s}$, and the velocity of the last 2500 epoch in E-direction is $2 \mathrm{~m} / \mathrm{s}$, the $\mathrm{N}$ direction involves two acceleration and deceleration movements accompanied by a change in direction, which is also in line with the trajectory on the experimental map. In the $U$ direction, the speed of the LS solution has obvious noise when the ship is making a turning motion, while the ARKF methods is relatively stable and with no obvious noise.
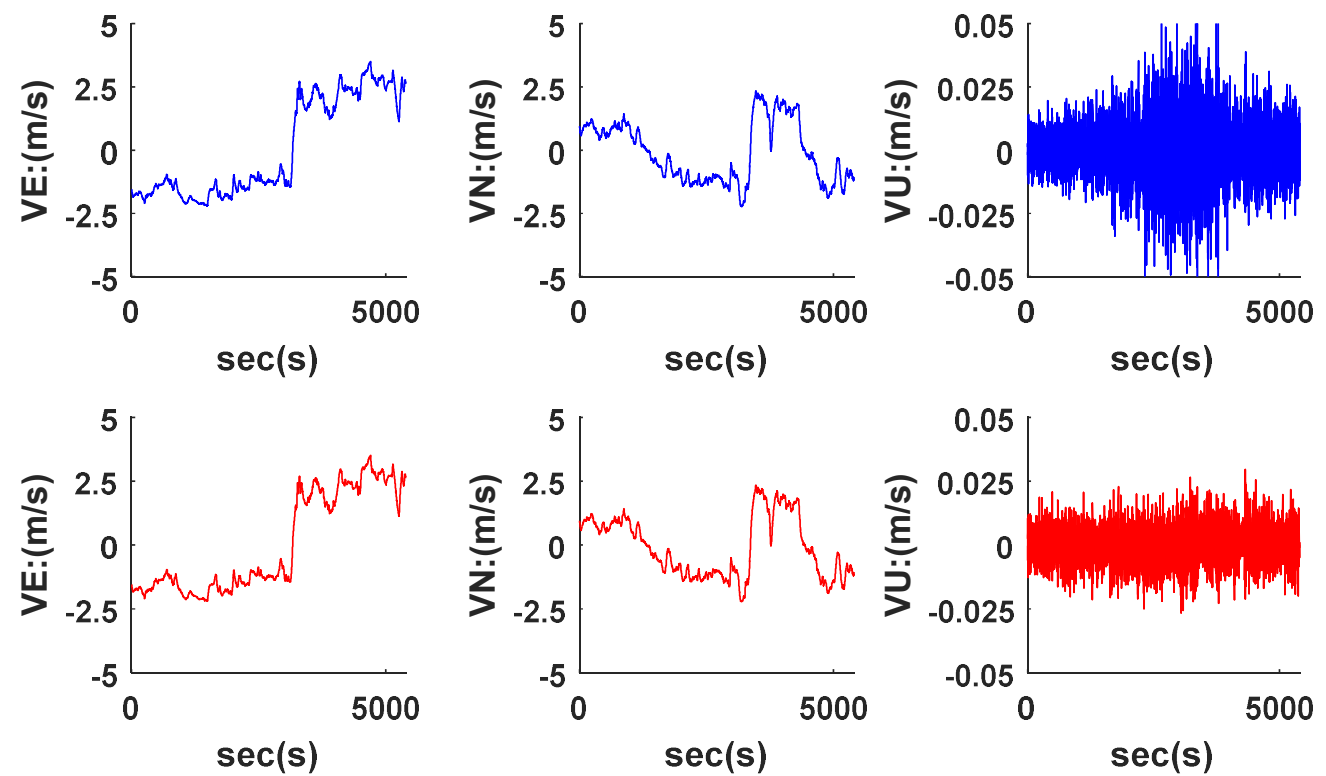

Figure. 5 The shipborne velocity in E/N/U three-direction calculated by the LS and ARKF methods. 

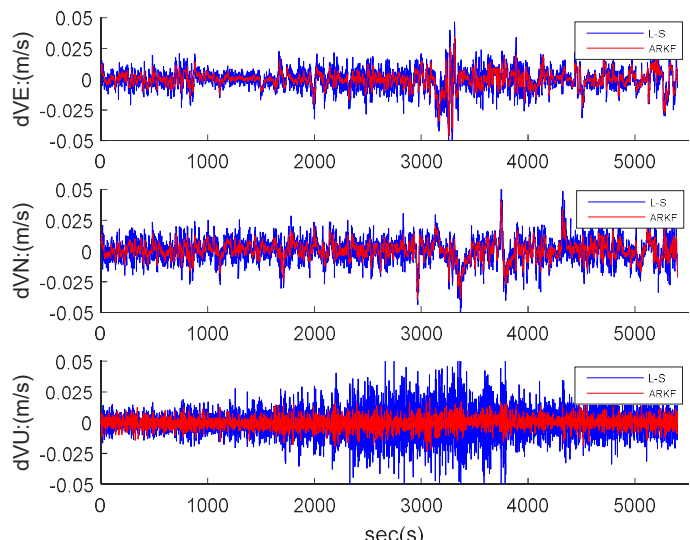

Figure.6 the figure of the velocity in $\mathrm{E} / \mathrm{N} / \mathrm{U}$ three-direction between tight combination of Internial Explorer (IE) with the L-S and ARKF methods.

As can be seen from Fig 6, the ARKF method has a relatively good denoising effect and changes steadily with the speed difference. However, the overall velocity calculated by LS method is relatively high in noise, with the mutual difference of the $\mathrm{E}$ and $\mathrm{N}$ direction up to $2.5 \mathrm{~cm} / \mathrm{s}$, while the $\mathrm{ARKF}$ method is within $1 \mathrm{~cm} / \mathrm{s}$. The LS solution in $U$ direction has the worst effect, with the noise up to $5 \mathrm{~cm} / \mathrm{s}$, while ARKF is stable within $2.5 \mathrm{~cm}$.

\begin{tabular}{cccccc}
\hline & & Max & Min & Mean & RMS \\
\hline L-s & VE & 0.0581 & 0.0000 & 0.0063 & 0.0088 \\
$(\mathrm{~m} / \mathrm{s})$ & VN & 0.0600 & 0.0000 & 0.0740 & 0.0098 \\
& VU & 0.1039 & 0.0000 & 0.0085 & 0.0118 \\
ARKF & VE & 0.0465 & 0.0000 & 0.0047 & 0.0067 \\
$(\mathrm{~m} / \mathrm{s})$ & VN & 0.0404 & 0.0000 & 0.0055 & 0.0074 \\
& VU & 0.0256 & 0.0000 & 0.0067 & 0.0088 \\
\hline
\end{tabular}

Table 2. Statistics of the cross-difference between the LS, ARKF and the IE velocity

We can know from the Tab.2, that the error mean of cross-differences between the velocity calculated by LS and IE in $\mathrm{N}, \mathrm{E}$ and $\mathrm{U}$ directions were $0.63 \mathrm{~cm} / \mathrm{s}, 0.74 \mathrm{~cm} / \mathrm{s}$ and 0.85 $\mathrm{cm} / \mathrm{s}$, respectively, while the ARKF was $0.47 \mathrm{~cm} / \mathrm{s}, 0.55 \mathrm{~cm} / \mathrm{s}$ and $0.67 \mathrm{~cm} / \mathrm{s}$, respectively. The RMS of the cross-differences in $\mathrm{E}, \mathrm{N}$ and $\mathrm{U}$ directions for $\mathrm{LS}$ method were $0.88 \mathrm{~cm} / \mathrm{s}$, $0.98 \mathrm{~cm} / \mathrm{s} 、 1.18 \mathrm{~cm} / \mathrm{s}$, while the ARKF method was $0.67 \mathrm{~cm} / \mathrm{s}$ 、 $0.74 \mathrm{~cm} / \mathrm{s} 、 0.88 \mathrm{~cm} / \mathrm{s}$. It was proved that the out Doppler velocity estimation by the ARKF method can also measure better when the ship is maneuvering. In view of the fact that the precision of the dynamic condition is lower than the static fact, it can be considered that the observation error of carrier phase accuracy in dynamic condition is lower than the static condition, and the observation environment is more complicated. The influence of the ionosphere and troposphere is more significant in the differential process, which is also consistent with the GNSS observation.

\section{CONCLUSION}

In this paper ,the processing results of static and dynamic data was analyzed which shows that compared with the solution of least squares, the ARKF method has higher accuracy and smoother, and the reliability of both plane and elevation directions is improved. From the analysis of the processing results of the shipborne GNSS/INS combined navigation dynamic observation data, with the GNSS/INS tight combination velocity by IE as the reference value, it can be seen that when the moving target state changes, the adaptive robust Kalman filter solution result is superior to the least squares solution result. The velocity deviation and the fluctuation amplitude is smaller, the velocity of points are more continuous, and the transition is more natural. The abnormal disturbance of the carrier observation can be controlled more effectively, and the precision of the filtering is improved which means that the method has a certain research value for solving problems such as observation abnormality and state abnormality. Therefore, the method can be applied to dynamic navigation and velocity measurement.

\section{ACKNOWLEDGEMENTS}

This research project was jointly supported by National key research and development plan of China (No.2016YFB0501705, No.2018YFF0212203, No. 2017YFC1405006), the key research and development plan of Shandong Province (No. 2018GHY115002)

\section{REFERENCES}

Zheng, K., Tang, L., 2017: Performance assessment of bds and gps/bds velocity estimation with stand-alone receiver. Journal of Navigation, 69(04), 869-882. doi.10.1017 /s0373463315000958

Wang, X., Liang, M., 2018: GPS Positioning Method Based on Kalman Filtering. 2018 International Conference on Robots \& Intelligent System (ICRIS). doi,10.1109/ICRIS.2018.00028

Min, L., He K., Xu T. , \& Biao, L. . (2018). Robust adaptive 
filter for shipborne kinematic positioning and velocity determination during the Baltic sea experiment. GPS Solutions, 22(3), 81-.doi, 10.1007/s10291-018-0747-5.

Gao S., Zhong Y., Li W., 2011: Random Weighting Method for Multisensor Data Fusion. IEEE Sensors Journal,2011, 11(9):1955-1961. doi,10.1109/JSEN.2011.2107896

Yang, Y., He, H. , Xu, G. , 2001: Adaptively robust filtering for kinematic geodetic positioning. Journal of Geodesy, 75(2-3), 109-116.doi: 10.1007/s001900000157.

Yang Y., Xu T, He H.,2001: On Adaptively Kinematic filtering //Selected papers for English of Acta Geodetica et Carto-graphica Sinica. The Mapping Publishing Company.

Yang, Y., Gao, W., 2006: An optimal adaptive kalman filter. Journal of Geodesy, 80(4), 177-183.doi, 10.1007/s00190-006-0041-0.

Cui. X. , Yang Y,2006:Adaptively robust filtering with classified adaptive factors. Progress in Natural Science, 16(8), 846-851.doi, 10.1080/10020070612330078.

Yang, Y., Xu, T. , 2003: An adaptive kalman filter based on sage windowing weights and variance components. Journal of Navigation, 56(2), 231-240.doi, 10.1017/s0373463303002248

Yang, Y., Gao, W., 2005: Influence comparison of adaptive factors on navigation results. Journal of Navigation,
58(3):471-478

Ren C., Ou J., Yunbin, Y., 2005: Application of adaptive filtering by selecting the parameter weight factor in precise kinematic gps positioning. Progress in Natural Science, 15(1), 41-46. doi, 10.1080/10020070512331341750

Xu, G. 2016: GPS: Theory, Algorithms and Applications, 2nd edition by Guochang Xu. Berlin: Springer, 2007.

He,K., 2015: Gnss kinematic position and velocity determination for airborne gravimetry. Acta Geodaetica et Cartographica Sinica. doi/10.11947/j.AGCS.2015.20150133

Tu, R.,2014: Fast determination of displacement by ppp velocity estimation. Geophysical Journal International, 196(3), 1397-1401.doi, 10.1093/gji/ggt480

PHILLIPS, Alan, H., 1984:Geometrical determination of pdop. Navigation, 31(4),

329-337.doi, 10.1002/j.2161-4296.1984.tb00883.x

Galili, U., Buehler, J., Shohet, S. B., \& Macher, B. A.,1987:The human natural anti-gal igg. iii. the subtlety of immune tolerance in man as demonstrated by crossreactivity between natural anti-gal and anti-b antibodies. Journal of Experimental Medicine, 165(3), 693-704.doi, 10.1084/jem.165.3.693

Yang, Y.X, 2006: Adaptive Navigation and Dynamic Positioning; Surveying and Mapping Press: Beijing, China 